\title{
Optimizing Transcutaneous Energy Transmitter Using Game Theory
}

\author{
Daniela Wolter Ferreira Touma ${ }^{1}$ and Luiz Lebensztajn ${ }^{2}$ \\ ${ }^{1}$ UNESP-Univ Estadual Paulista, Guaratinguetá SP-12516-410, Brazil \\ ${ }^{2}$ Escola Politécnica da Universidade de São Paulo, São Paulo 05508-900, Brazil
}

\begin{abstract}
Over the past recent years, several studies, seeking reliable Transcutaneous Energy Transmitters (TETs), have used different types of optimization techniques with different objectives. Algorithms with multiple objectives and constraints resulted in different configurations in the Pareto front, making the decision of the final configuration harder. Moreover, the game theory has been gaining a lot of ground in the engineering design, mainly in decision making in the optimization problems. This study uses the advantage of the game theory together with genetic algorithm to find one final configuration of TET, which fulfills the specified constraints while optimizing different objective functions. It uses three players playing with six different variables, which were assigned to each player in a strategic manner according to each player's target.
\end{abstract}

Index Terms-Finite element method (FEM), game theory, inductive power transmission, optimal design.

\section{INTRODUCTION}

$\mathbf{T}$ RANSCUTANEOUS energy transmitters (TETs) are systems widely studied by several researchers with the aim of transferring energy inside the body without percutaneous electrical leads passing through the skin that can cause infection. TETs normally use an inductive link between a primary coil external to the body and a secondary coil underneath the skin, similar to a coreless transformer [1], [2]. Since they perform such an important task, seeking the smallest coils that transfer the necessary power at the maximum efficiency has attracted attention over recent years. This search was the goal of [3], which used multiobjective genetic algorithm (MGA) optimization, having seven final different configurations in the Pareto front $(\mathrm{PF})$. The problem has become to decide which of the configurations from the PF should be used. In recent years, new kinds of optimization methods have been investigated, and the game theory is among them. In actuality, the game theory is not categorized into an optimization method in an engineering field, but categorized into a decision-making method in economics. Nevertheless, it was used in the multiobjective optimal design problems of electromagnetic apparatus [4]-[6].

For more practical use of an optimization shape design method, it is necessary to optimize several objectives, and the result to be obtained has to be rational from the viewpoints of engineering, robustness, and productivity. In this paper, a method utilizing the game theory is proposed for obtaining the smallest TET coils with the maximum transmission efficiency and the least coil heating possible that supply the necessary power at the required voltage ranges. Unlike the MGA, this approach obtains one single solution.

Manuscript received July 6, 2015; revised October 9, 2015; accepted October 21, 2015. Date of publication October 27, 2015; date of current version February 17, 2016. Corresponding author: D. Wolter Ferreira Touma (e-mail: daniwolter@feg.unesp.br).

Color versions of one or more of the figures in this paper are available online at http://ieeexplore.ieee.org.

Digital Object Identifier 10.1109/TMAG.2015.2495197

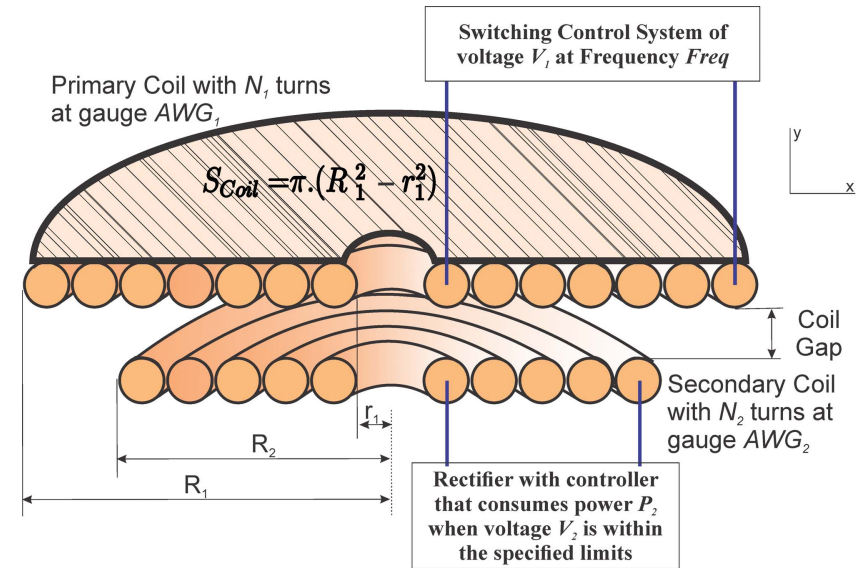

Fig. 1. Representation of the TET system.

\section{TRAnScutaneous EnERgy TRAnsmitTER OPTIMIZATION}

\section{A. Transcutaneous Energy Transmitter}

Fig. 1 shows the TET system to be optimized. It is composed of two coils: one that transmits the power (primary coil) with $N_{1}$ turns of gauge $\mathrm{AWG}_{1}$ and another that receives the power (secondary coil) with $N_{2}$ turns of gauge $\mathrm{AWG}_{2}$. The primary coil is supplied with voltage $V_{1}$ at frequency Freq from a switching control system. The secondary coil uses the received power to feed a rectifier with a controller that supplies power to an artificial organ. The system composed of the rectifier, controller, and artificial organ consumes constant power $P_{2}$ when the received voltage $V_{2}$ is within some specified limits.

The behavior of this system $\left(P_{2}\right.$ and $\left.V_{2}\right)$ can be virtually obtained by the finite-element method (FEM) when supplied the input information, such as $N_{1}$ turns of gauge $\mathrm{AWG}_{1}$, $N_{2}$ turns of gauge $\mathrm{AWG}_{2}$, voltage $V_{1}$ at frequency Freq, and among other characteristics of the coils. Hence, the geometry was implemented in the software Gmsh [7] and its FE equations simulated by the software GetDP [8], 
which allows easy interface with MATLAB to implement an optimization method.

\section{B. Parameters of the Optimization}

Similar to [3], in this paper, the increase of temperature is indirectly accounted for by the relation of the dissipated power $\left(P_{\text {dissipated }}\right)$ in $\mathrm{W}$ to the coil area $S_{\text {Coil }}$ (see Fig. 1) in $\mathrm{m}^{2}$, denominated "thermo factor" $(\lambda)$ in $\mathrm{W} / \mathrm{m}^{2}$

$$
\lambda=P_{\text {dissipated }} / S_{\text {Coil }} \text {. }
$$

It is desired to find the smallest TET coils with the smallest "thermo factor" that supplies the required power at the required voltage limits for a range of specified coupling situations. Moreover, the efficiency of the transmission $(\eta)$ should also be consider to avoid unnecessary losses during the transmission and to allow more efficient electronic circuits to supply and to receive power to and from the TET coils. However, the "thermo factor" and the transmission efficiency depend on the coil gap (see Fig. 1). This would make an extremely large number of objective functions unless these objective functions are grouped together, such as

$$
\left\{\begin{array}{l}
f_{1}=\sqrt{\sum_{i}\left(\lambda_{1, i}^{2}\right)} ; \quad f_{2}=\mathrm{Vol}_{1} \\
f_{3}=\sqrt{\sum_{i}\left(\lambda_{2, i}^{2}\right)} ; \quad f_{4}=\mathrm{Vol}_{2} \\
f_{5}=100-\operatorname{mean}\left(\eta_{i}\right) .
\end{array}\right.
$$

In (2), Vol is the coil volume in $\mathrm{cm}^{3}, \lambda$ is the coil "thermo factor" in $\mathrm{W} / \mathrm{m}^{2}, \eta$ is the efficiency in \%, the subscripts 1 and 2 represent the primary and secondary coils, respectively, and the subscript $i$ is the coil gap index at which the TET was simulated $(4,8,12,16,20$, and $25 \mathrm{~mm})$.

The TET parameters that could help to meet the demands of this project (variables of decision of the optimization) are described in Section II-A $\left(N_{1}, N_{2}, \mathrm{AWG}_{1}, \mathrm{AWG}_{2}, V_{1}\right.$, and Freq). Note that the objective functions $f_{2}$ and $f_{4}$ depend only on the variables $N_{1}, \mathrm{AWG}_{1}$ and $N_{2}, \mathrm{AWG}_{2}$, respectively. However, all other functions depend on all six variables of decision.

Guaranteeing that the TET will always be able to supply the required power $\left(P_{2}\right)$ at the right range of voltage to the internal system $\left(V_{2}\right)$ at the specified distances is of extreme importance. Thus, the transfer power and voltage must be mandatory constraints at each distance between coils from 4 to $25 \mathrm{~mm}$, totalizing 18 constraints as

$$
\text { s.t. }\left\{\begin{array}{l}
g_{1, i}=19-P_{2, i}<0 \\
g_{2, i}=8-V_{2, i}<0 \\
g_{3, i}=V_{2, i}-28<0
\end{array} \quad i=1, \ldots, 6\right.
$$

where $i$ is an index from 1 to 6 that represents each distance between coils $(4,8,12,16,20$, and $25 \mathrm{~mm})$. These non-linear constraints allow a more reliable TET operation.

\section{OPTIMIZATION OF TET}

\section{A. Game-Theory Optimization}

The game theory, proposed in [9], mathematically solves the decision-making problem, where a solution of each objective function can influence the outcomes of the others. It uses an abstract mathematical model of a multiagent decision-making setting with the goal to include all and only those aspects that are relevant to the decisions that players must make [10].

For an optimization problem with $n$ objectives functions, the game theory consists in having $n$ players, each optimizing its own objective function given that all the other criteria are fixed by the other players. Thus, each player must consider how every other player will act to make an optimal choice.

In order to have a fair game, each player should be responsible for variables that affect not only its objective function but also all the objective functions of the other players, even with their variables fixed. Then, the game has a sequence starting with one player, which seeks its variables to find the most optimized single objective function of its responsibility. This optimization can be performed by single genetic algorithm (GA) with the necessary constraints. As soon as this player finds its best solution, the next player starts its turn. Then, the latter optimizes its objective function with the constraints while using the fixed variables obtained from the previous player and the consecutive players. In this way, each player runs its GA optimization on its own turn, finding the variables that best fit its objective function before allowing the turn of the next player. When the last player finds its solution, the next player is again the first one, and another round of the game starts.

If, in the turn, the sought variables are the same as they were in the previous round, the game considers that this player has skipped its turn, since there is no improvement in its criterion. However, in the next round, this player may find a new solution if the other players had changed their variables.

When no player can further improve its criterion, i.e., all the players skip their turns on the round, it means that the game has reached a state of equilibrium called Nash Equilibrium (NE), which is the most famous example of a solution concept.

Indeed, the NE is a selection of choices for players such that no player would prefer to unilaterally deviate from this selection. A different solution could be better only if a new game plays with different strategies and/or conditions.

\section{B. Convergence}

The proposed approach to find the NE is based on the following hypothesis: each player can minimize his own function $\left(f^{i}\right)$ by dealing with a subset of the optimization variables $\left(x_{j}\right), j=1, \ldots, M^{i}$. The NE is then found when all the players could not improve its solution. The minimization of each function $f^{i}(x)$ could be constrained or not. Thus, the proposed solution is sequential and depends mainly on the starting point, the order of the players, and the nature of functions, i.e., the solution of the problem could even suffer convergence problems. To illustrate the convergence of the proposed method, some analytical problems were analyzed.

The first analytical problem is the ZDT6 function, proposed in [11]. This function has ten optimization variables, $0 \leq x_{i} \leq 1$, has no constraints and is described as follows:

$$
\left\{\begin{array}{l}
f_{1}\left(x_{1}\right)=1-\exp \left(-4 x_{1}\right) \cdot \sin ^{6}\left(6 \pi x_{1}\right) \\
f_{2}=g(x) \times h\left(f_{1}, g\right)
\end{array}\right.
$$




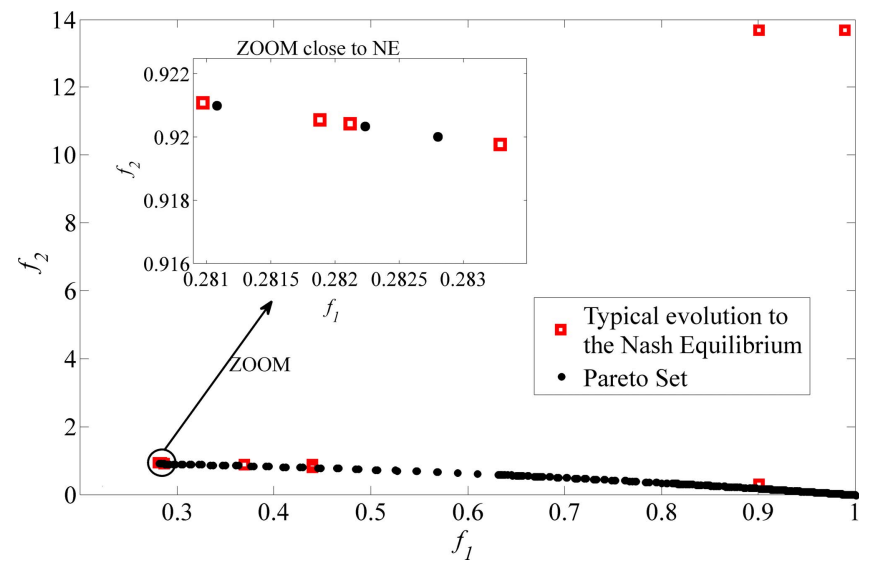

Fig. 2. Evolution of the ZDT6 function.

where

$$
\left\{\begin{array}{l}
g\left(x_{2}, \ldots, x_{m}\right)=1+9 \cdot\left(\sum_{i=2}^{m} x_{i}\right)^{0.25} \\
h\left(f_{1}, g\right)=1-\left(f_{1} / g\right)^{2} .
\end{array}\right.
$$

The second analyzed function is the Osyczka and Kundu function [12], which can be described as

$$
\begin{gathered}
\left\{\begin{aligned}
\begin{array}{l}
f_{1}(x)=- \\
{\left[25 .\left(x_{1}-2\right)^{2}+\left(x_{2}-2\right)^{2}+\left(x_{3}-1\right)^{2}\right.} \\
\left.\quad+\left(x_{4}-4\right)^{2}+\left(x_{5}-1\right)^{2}\right]
\end{array} \\
f_{2}(x)=\sum_{i=1}^{6} x_{i}^{2}
\end{aligned}\right. \\
\text { s.t. } \begin{cases}g_{1}(x)=x_{1}+x_{2}-2 \geq 0 ; & g_{4}(x)=2-x_{1}+3 \cdot x_{2} \geq 0 \\
g_{2}(x)=6-x_{1}-x_{2} \geq 0 ; & g_{5}(x)=4-\left(x_{3}-3\right)^{2}-x_{4} \geq 0 \\
g_{3}(x)=2+x_{1}-x_{2} \geq 0 ; & g_{6}(x)=\left(x_{5}-3\right)^{2}+x_{6}-4 \geq 0 .\end{cases}
\end{gathered}
$$

Fig. 2 shows a typical evolution of the ZDT6 function. For this function, the association between function and parameters is unique: $f_{1}$ is linked to $x_{1}$ and $f_{2}$ is linked to $x_{i}$, $2 \leq i \leq 10$, and the NE $\left(f_{1}=0.281\right.$ and $\left.f_{2}=0.921\right)$ is always found if the setup of GA is carefully done. The main challenge for this function is that $f_{1}$ and $f_{2}$ are very low sensitive to variations on the parameters when close to the NE. Hence, there could be a lack of convergence, because GA could trap due to the adopted tolerance function. This drawback shows one of the limitations of this method. The zoomed-in view of Fig. 2 clarifies the behavior of this function.

The game theory was also applied to the Osyczka and Kundu function four times with two players using different variables (among $x_{1}$ to $x_{6}$ ) with different starting points and configurations, which depend on which variable each player is using. Table I shows the result of each game (IDs), where the index of the variable on column $f_{1}$ and $f_{2}$ indicates which variable was used by the players. Some NE points belong to the Pareto set, but there are some points that are non-dominated (ND) and others are dominated (D), i.e., an NE does not always belong to the Pareto set. Moreover, the dependence on the choice of optimization variables is an another limitation of the method observed in Table I.
TABLE I

RESULTS From THE OSYCZKA AND KUndU FUnCTION

\begin{tabular}{cccccccccccc}
\hline \hline & Player 1 & Player 2 & & & & & & & & & \\
ID & $f_{1}$ & $f_{2}$ & $x_{1}$ & $x_{2}$ & $x_{3}$ & $x_{4}$ & $x_{5}$ & $x_{6}$ & $P / N P$ & $f_{1}$ & $f_{2}$ \\
\hline 1 & $x_{1} \rightarrow x_{5}$ & $x_{6}$ & 0 & 2 & 5 & 0 & 5 & 0 & $\mathrm{D}$ & -148 & 54 \\
2 & $x_{1} \rightarrow x_{5}$ & $x_{6}$ & 5 & 1 & 5 & 0 & 1 & 0 & ND & -274 & 76 \\
3 & $x_{1} \rightarrow x_{2}$ & $x_{3} \rightarrow x_{6}$ & 0 & 2 & 1 & 0 & 1 & 0 & ND & -116 & 6 \\
4 & $x_{1} \rightarrow x_{3}$ & $x_{4} \rightarrow x_{6}$ & 0 & 2 & 5 & 0 & 1 & 0 & $\mathrm{D}$ & -132 & 30 \\
\hline \hline
\end{tabular}

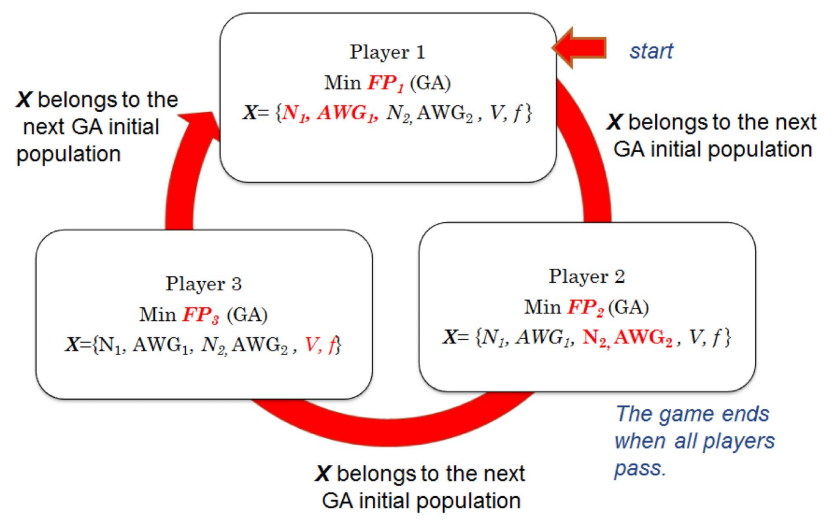

Fig. 3. Sequential game structure of the TET.

In this case, it is interesting to observe that all NE points of Table I are active constraint $\left[g_{i}(x)=0\right]$. This dependence of the choice of parameters and the observation that the NE could (or not) belong to the Pareto set is also reported in [5].

\section{Game Theory Applied in the Optimization of TET}

The objective of the game is to minimize the "thermo factor" and the volume simultaneously in both coils while maximizing efficiency in transmitting the required power.

According to [6], each objective function related to each player depends on all the variables of decision, i.e., it depends on the variable controlled by its player as well as on all the other variables controlled by the other players, which are fixed at the turn of the specific player. At this point of view, $f_{2}$ in (2) is not a good function to be adopted as an objective of a player because it does not depend on $N_{2}, \mathrm{AWG}_{2}, V_{1}$, and Freq. The same applies for $f_{4}$ in (2). Hence, a game with five players, each with one objective function, would not work well.

Therefore, three players were considered in this game: 1) player $1\left(P_{1}\right)$ deals with the primary coil, i.e., $P_{1}$ controls the variables of decision of the primary coil $\left(N_{1}\right.$ and $\mathrm{AWG}_{1}$ ) to minimize the progression of the "thermo factor" and volume of the primary coil; 2) player $2\left(P_{2}\right)$ deals with the secondary coil, i.e., $P_{2}$ controls the variables of decision of the secondary coil $\left(N_{2}\right.$ and $\left.\mathrm{AWG}_{2}\right)$ to minimize the progression of the "thermo factor" and volume of the secondary coil; and 3$)$ player $3\left(P_{3}\right)$ controls the TET power supply capability, i.e., the general variables of decision (Freq and $V_{1}$ ) to maximize the efficiency $(\eta)$. Since player 1 and player 2 deal with two objective functions of different kinds of nature, a normalization of these functions was performed in order to allow the addition between them. Thus, the objective 
TABLE II

DEVELOPMENT OF THE GAME

\begin{tabular}{|c|c|c|c|c|c|c|c|c|c|c|c|c|c|c|c|}
\hline & \multicolumn{8}{|c|}{ Variables } & \multicolumn{7}{|c|}{ Objective functions } \\
\hline & Iteration & $\begin{array}{c}N_{l} \\
\text { [turns] }\end{array}$ & $A W G_{1}$ & $\begin{array}{c}N_{2} \\
\text { [turns] }\end{array}$ & $A W G_{2}$ & $\begin{array}{c}\text { Freq } \\
{[\mathrm{kHz}]}\end{array}$ & $\begin{array}{c}V_{1} \\
{[\mathrm{~V}]}\end{array}$ & $F P_{1}$ & $\begin{array}{c}\lambda_{1} \\
{\left[\mathrm{~W} / \mathrm{m}^{2}\right]}\end{array}$ & $\begin{array}{c}\mathrm{Vol}_{1} \\
{\left[\mathrm{~cm}^{3}\right]}\end{array}$ & $F P_{2}$ & $\begin{array}{c}\lambda_{2} \\
{\left[\mathrm{~W} / \mathrm{m}^{2}\right]}\end{array}$ & $\begin{array}{c}\mathrm{Vol}_{2} \\
{\left[\mathrm{~cm}^{3}\right]}\end{array}$ & $\begin{array}{c}F P_{3}[\%] \\
100-\eta\end{array}$ & \\
\hline Start & 0 & 60 & 20 & 23 & 25 & 144 & 25 & - & 279.04 & 8.533 & - & 52.27 & 0.406 & 57.75 & \\
\hline \multirow{3}{*}{$\begin{array}{l}I \\
I \\
I\end{array}$} & 1 & 60 & 22 & 23 & 25 & 144 & 25 & 1.54 & 277.96 & 4.617 & 2.02 & 53.54 & 0.406 & 50.99 & $\mathrm{C}$ \\
\hline & 2 & 60 & 22 & 33 & 31 & 144 & 25 & 1.98 & 273.17 & 4.617 & 0.75 & 19.56 & 0.156 & 50.82 & $\mathrm{C}$ \\
\hline & 3 & 60 & 22 & 33 & 31 & 177 & 27 & 1.86 & 233.96 & 4.617 & 2.66 & 32.38 & 0.156 & 46.43 & $\mathrm{C}$ \\
\hline \multirow{3}{*}{$\begin{array}{l}P \\
P \\
P\end{array}$} & 4 & 60 & 22 & 33 & 31 & 177 & 27 & 2.00 & 233.96 & 4.617 & 2.00 & 32.38 & 0.156 & 46.43 & $\mathrm{~S}$ \\
\hline & 5 & 60 & 22 & 34 & 31 & 177 & 27 & 2.02 & 239.39 & 4.617 & 1.83 & 25.36 & 0.163 & 46.56 & C \\
\hline & 6 & 60 & 22 & 34 & 31 & 437 & 36 & 1.66 & 158.34 & 4.617 & 4.30 & 83.76 & 0.163 & 33.95 & $\mathrm{C}$ \\
\hline \multirow{3}{*}{$\begin{array}{l}P_{1} \\
P_{2} \\
P_{3} \\
\end{array}$} & 7 & 60 & 22 & 34 & 31 & 437 & 36 & 2.00 & 158.34 & 4.617 & 2.00 & 83.76 & 0.163 & 33.95 & $\mathrm{~S}$ \\
\hline & 8 & 60 & 22 & 34 & 31 & 437 & 36 & 2.00 & 158.34 & 4.617 & 2.00 & 83.76 & 0.163 & 33.95 & $\mathrm{~S}$ \\
\hline & 9 & 60 & 22 & 34 & 31 & 437 & 36 & 2.00 & 158.34 & 4.617 & 2.00 & 83.76 & 0.163 & 33.95 & $\mathrm{~S}$ \\
\hline
\end{tabular}

The last column indicates $(\mathrm{C})$ if the player changed its configuration to get a better criterion or (S) if it skipped its turn, i.e., it can no further improve its criterion.

function of each player is

$$
\begin{cases}\mathrm{FP}_{1}=f_{1} / f_{1 \text { old }}+f_{2} / f_{2 \text { old }} & \text { for player } 1 \\ \mathrm{FP}_{2}=f_{3} / f_{3 \text { old }}+f_{4} / f_{4 \text { old }} & \text { for player } 2 \\ \mathrm{FP}_{3}=100-\eta & \text { for player } 3 .\end{cases}
$$

The functions used by all players $\left(f_{1}, f_{2}, f_{3}, f_{4}\right.$, and $\left.f_{5}\right)$ are defined in (2); $f_{i \text { old }}$ is the value of the function $i$ at the previous iteration. Each player uses the GA to minimize its objective function with the constraints defined by (3). Fig. 3 shows the sequential structure of the game theory applied with GA for the TET problem. Note that, the game starts with an initial configuration, which is part of the initial population of the GA. Then, one by one, each player optimizes its objective function by varying its controlled variables, while the variables of the other players remain fixed at their previous values.

\section{RESUlts}

The development of the game applied in this paper is shown in Table II. The game was composed of three rounds after the starting configuration $\left(N_{1}=60\right.$ turns, $N_{2}=23$ turns, $\mathrm{AWG}_{1}=20, \mathrm{AWG}_{2}=25, V_{1}=25 \mathrm{~V}$, and Freq $=144 \mathrm{kHz}$ ). The configurations in all the tries do not violate any constraint. At each round, the target of each player either improved or worsened depending on who was playing. However, at the final round, each player could not find any other variables that could improve its target, thus reaching equilibrium with the final configuration $\left(N_{1}=60\right.$ turns, $N_{2}=34$ turns, $\mathrm{AWG}_{1}=$ $22, \mathrm{AWG}_{2}=31, V_{1}=36 \mathrm{~V}$, and Freq $=437 \mathrm{kHz}$ ). The selected configuration improved all the functions except for the "thermo factor" on the secondary coil. In addition, increasing the coil gap decreases the load voltage from 38.4 to $12.9 \mathrm{~V}$ and the transmission efficiency from $73.9 \%$ to $56 \%$, while supplying the constant power of $19 \mathrm{~W}$, what shows a reliable TET design.

\section{CONCLUSION}

This paper proposed the use of game theory to solve the decision-making problem of the multiobjective optimization of TETs. The variables for each player were assigned in a physical matter, whereas player 1 was responsible for the variables of the primary coil, player 2 the secondary coil, and player 3 the transmission variables. The same philosophy can be applied with different variables distribution. This paper also used a sequential structure starting with player 1 , then 2 , and 3 . For future works, the variable distribution and the player sequence should be analyzed in relation to the convergence.

With the proposed relation variables/objective functions and sequence, the game theory guaranteed that all the constraints were not violated and achieved a solution better than the starting configuration with less FEM simulations than the MGA.

\section{ACKNOWLEDGMENT}

This work was supported in part by the Fundação de Amparo à Pesquisa do Estado de São Paulo under Grant 11/18341-3 and Grant 15/03949-7, and in part by the Conselho Nacional de Desenvolvimento Científico e Tecnológico under Grant 309350/2012-3.

\section{REFERENCES}

[1] H. Miura, S. Arai, Y. Kakubari, F. Sato, H. Matsuki, and T. Sato, "Improvement of the transcutaneous energy transmission system utilizing ferrite cored coils for artificial hearts," IEEE Trans. Magn., vol. 42, no. 10 , pp. $3578-3580$, Oct. 2006.

[2] H. Matsuki, M. Shiiki, K. Murakami, and T. Yamamoto, "Investigation of coil geometry for transcutaneous energy transmission for artificial heart," IEEE Trans. Magn., vol. 28, no. 5, pp. 2406-2408, Sep. 1992.

[3] D. W. Ferreira, L. Lebensztajn, L. Krähenbühl, F. Morel, and C. Vollaire, "A design proposal for optimal transcutaneous energy transmitters," IEEE Trans. Magn., vol. 50, no. 2, Feb. 2014, Art. ID 7024704.

[4] T. Miyamoto, S. Noguchi, and H. Yamashita, "Selection of an optimal solution for multiobjective electromagnetic apparatus design based on game theory," IEEE Trans. Magn., vol. 44, no. 6, pp. 1026-1029, Jun. 2008.

[5] M. Cioffi, P. Di Barba, A. Formisano, and R. Martone, "Pareto optima and Nash equilibria: An effective approach to the shape design in electromagnetics," COMPEL-Int. J. Comput. Math. Elect. Electron. Eng., vol. 27, no. 4, pp. 845-854, 2008.

[6] P. Di Barba, "Strategies of game theory for the automated optimal design in electromechanics," Int. J. Appl. Electromagn. Mech., vol. 27, no. 4, pp. $275-295,2008$.

[7] C. Geuzaine and J.-F. Remacle, "Gmsh: A 3-D finite element mesh generator with built-in pre- and post-processing facilities," Int. J. Numer. Methods Eng., vol. 79, no. 11, pp. 1309-1331, 2009.

[8] P. Dular and C. Geuzaine. GetDP: A General Environment for the Treatment of Discrete Problems. [Online]. Available: http://geuz.org/getdp/, accessed Dec. 2014.

[9] J. Nash, "Non-cooperative games," Ann. Math., vol. 54, no. 2, pp. 286-295, 1951.

[10] M. Wooldridge, "Does game theory work?" IEEE Intell. Syst., vol. 27, no. 6, pp. 76-80, Nov./Dec. 2012.

[11] E. Zitzler, K. Deb, and L. Thiele, "Comparison of multiobjective evolutionary algorithms: Empirical results," Evol. Comput., vol. 8, no. 2, pp. 173-195, 2000.

[12] A. Osyczka and S. Kundu, "A new method to solve generalized multicriteria optimization problems using the simple genetic algorithm," Struct. Optim., vol. 10, no. 2, pp. 94-99, 1995. 